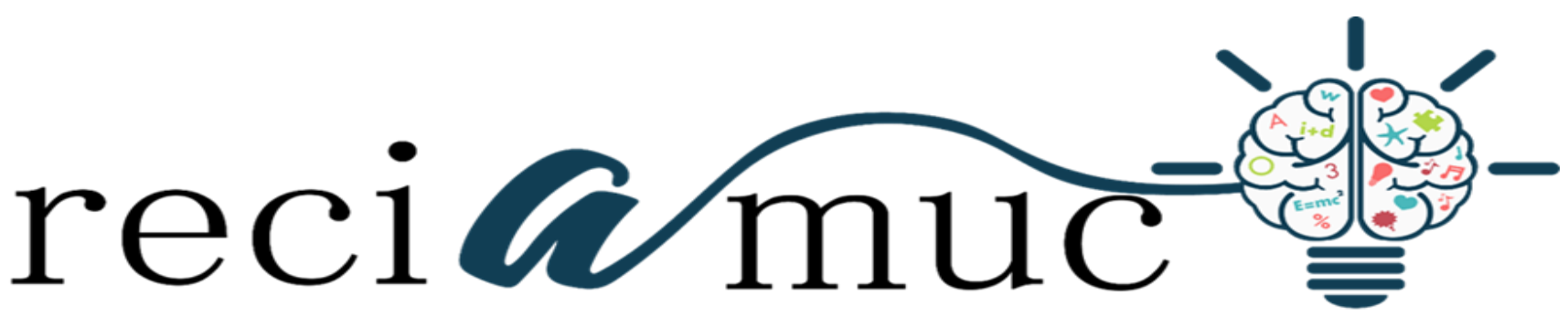

Revista cientifica de investigación actualización del mundo de las ciencias

Andrea Enriqueta Naranjo Castillo a ; Shyrle Lisbeth Vaca Rodríguez ${ }^{\text {b; }}$ María

Gabriela Lucin Salazar ${ }^{\text {c; }}$ Danilo José Cabrera Galecio ${ }^{\mathrm{d}}$

Síndrome mediastinal causas, diagnóstico y tratamiento

Mediastinal syndrome causes, diagnosis and treatment

Revista Científica de Investigación actualización del mundo de las Ciencias. Vol. 3 núm., 1, enero, ISSN: 2588-0748, 2018, pp. 242-271

DOI: $10.26820 /$ reciamuc/3.(1).enero.2019.242-271

URL: $\underline{\text { http://reciamuc.com/index.php/RECIAMUC/article/view/234 }}$

Código UNESCO: 3205 Medicina Interna

Tipo de Investigación: Artículo de Revisión

Editorial Saberes del Conocimiento

Recibido: 10/12/2018

Aceptado: 20/01/2019

Publicado: 30/01/2019

Correspondencia: director@ recumauc.com

\footnotetext{
a. Diploma Superior en Atención Primaria en Salud; Médico; Guayaquil, Ecuador.

b. Médico; Guayaquil, Ecuador.

c. Médico; Guayaquil, Ecuador.

d. Médico; Guayaquil, Ecuador.
} 


\section{Síndrome mediastinal causas, diagnóstico y tratamiento}

Vol. 3, núm. 1., (2019)

Andrea Enriqueta Naranjo Castillo; Shyrle Lisbeth Vaca Rodríguez; María Gabriela Lucin

Salazar; Danilo José Cabrera Galecio

\section{RESUMEN}

El mediastino corresponde a una parte del cuerpo humano que contienen los principales organismos del tórax, las cuales delimita entre el aparato digestivo, la columna vertebral y esternón. Las enfermedades pueden alterar el funcionamiento de los demás órganos que hacen vida dentro del mediastino debido a que modifican el equilibrio estático y dinámico del entorno. El síndrome mediastinal se caracteriza por ejercer una compresión en los diferentes órganos del mediastino a través de una masa que puede ser benigna o maligna según sea el caso del paciente. Poder encontrar las causas y su diagnóstico permitirá que el tratamiento sea el más acorde a la mejora en la salud del paciente. Por lo tanto, esta investigación tiene como objetivo general analizar las causas, diagnóstico y tratamiento del síndrome mediastinal. La metodología empleada se basó en una investigación de carácter documental y bibliográfico. Los resultados arrojaron que hay diferentes patologías dentro del síndrome mediastinal las cuales son el síndrome de la vena cava superior, el síndrome de la vena cava inferior, linfoma, neuroblastomas y neurfibromas. La conclusión arrojó que los síntomas generales son tos, disnea y dolor torácico. Las causas son diversas y dependen de cada una de las patologías. El hallazgo de estas enfermedades ocurre de manera incidental. Dependiendo de la histología del paciente se aplican radiografías, TCMD, resonancia magnética o gammagrafías como técnicas diagnósticas. El tratamiento predominante es extracción quirúrgica o aplicación de quimioterapia o radioterapia según sea el caso, lo cual va permitir una mejor calidad de vida del paciente y sus familiares.

Palabras Claves: Mediastino; Síndrome Mediastinal; Diagnóstico; Tratamiento. 


\section{Síndrome mediastinal causas, diagnóstico y tratamiento}

Vol. 3, núm. 1., (2019)

Andrea Enriqueta Naranjo Castillo; Shyrle Lisbeth Vaca Rodríguez; María Gabriela Lucin

Salazar; Danilo José Cabrera Galecio

\section{ABSTRACT}

The mediastinum corresponds to a part of the human body that contains the main organisms of the thorax, which delimits between the digestive system, the spine and sternum. Diseases can alter the functioning of other organs that make life within the mediastinum because they modify the static and dynamic equilibrium of the environment.The mediastinal syndrome is characterized by exerting a compression on the different organs of the mediastinum through a mass that can be benign or malignant depending on the case of the patient. Being able to find the causes and their diagnosis will allow the treatment to be the most appropriate to the improvement of the patient's health. Therefore, this research has as its general objective to analyze the causes, diagnosis and treatment of the mediastinal syndrome. The methodology used was based on a documentary and bibliographic research. The results showed that there are different pathologies within the mediastinal syndrome which are the superior vena cava syndrome, the inferior vena cava syndrome, lymphoma, neuroblastomas and neurofibromas. The conclusion showed that the general symptoms are cough, dyspnea and chest pain. The causes are diverse and depend on each of the pathologies. The finding of these diseases occurs incidentally. Depending on the histology of the patient, X-rays, MDCT, magnetic resonance or scintigraphy are used as diagnostic techniques. The predominant treatment is surgical removal or application of chemotherapy or radiotherapy as the

Key Words: Mediastinum; Mediastinal Syndrome; Diagnosis; Treatment. 


\section{Síndrome mediastinal causas, diagnóstico y tratamiento}

Vol. 3, núm. 1., (2019)

Andrea Enriqueta Naranjo Castillo; Shyrle Lisbeth Vaca Rodríguez; María Gabriela Lucin Salazar; Danilo José Cabrera Galecio

\section{Introducción.}

Para poder comprender el síndrome mediastinal es necesario analizar la importancia que radica el mediastino en la salud del ser humano. El mediastino es el espacio comprendido entre el orificio torácico superior arriba, el diafragma abajo, el esternón enfrente, la columna vertebral atrás y la pleura mediastinal a los lados, excepto los pulmones, todas las vísceras torácicas se hallan en el mediastino(Ibarra-Pérez, Kelly-García, \& Fernández-Corzo, 2001). Esto se puede observar en la Figura 1.

(A)

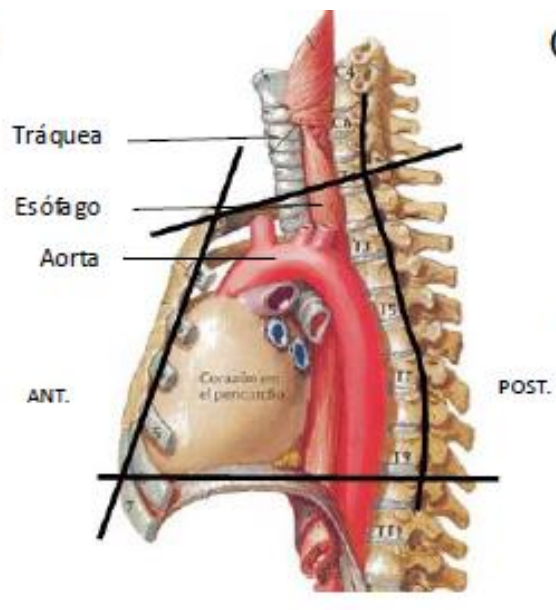

(B)

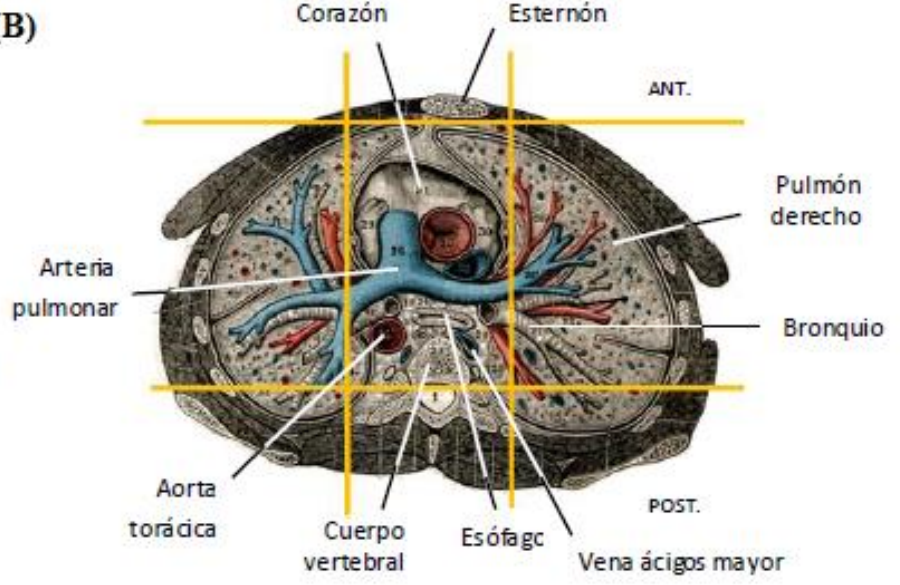

Figura 1. Límites del mediastino. (A) Vista lateral y (B) Vista transversal

Fuente: (Tramontini, 2019)

Las partes que conforman el mediastino tienen una importancia porque el espacio generado cumple una función de mantener el equilibrio estático y dinámico y a su vez permite la circulación venosa del entorno. 


\section{Síndrome mediastinal causas, diagnóstico y tratamiento}

Vol. 3, núm. 1., (2019)

Andrea Enriqueta Naranjo Castillo; Shyrle Lisbeth Vaca Rodríguez; María Gabriela Lucin Salazar; Danilo José Cabrera Galecio

La base del mismo es el diafragma, que presenta diversos orificios para el paso de estructuras vasculares y digestivas. El límite anterior se encuentra en el esternón y las articulaciones esternocondrales, y el posterior en la columna vertebral y las articulaciones costovertebrales. El extremo comunica con el cuello a través del desfiladero cérvico-torácico (Heras Gómez, 2006, pág. 1231).

Ahora las enfermedades que empezaron a surgir dentro de esta región permitieron clasificar el mediastino en 3 zonas con el fin de poder localizar, diagnósticas y tratar estas enfermedades. En la Figura 2 se puede detallar dos de estas partes.

El mediastino se divide en tres porciones: anterosuperior, media y posterior. Según esta división el mediastino anterosuperior incluye el timo, el cayado aórtico y sus ramas, el tronco venoso braquiocefálico y tejido linfático; el mediastino medio incluye corazón, pericardio, ganglios linfáticos, estructuras nerviosas, tráquea e hilio pulmonar y el mediastino posterior contiene el esófago, la aorta, ganglios autónomos paravertebrales, cadena simpática, nervio vago, conducto torácico y vena ácigos(Patané, y otros, 2006, pág. 47). 


\section{Síndrome mediastinal causas, diagnóstico y tratamiento}

Vol. 3, núm. 1., (2019)

Andrea Enriqueta Naranjo Castillo; Shyrle Lisbeth Vaca Rodríguez; María Gabriela Lucin Salazar; Danilo José Cabrera Galecio

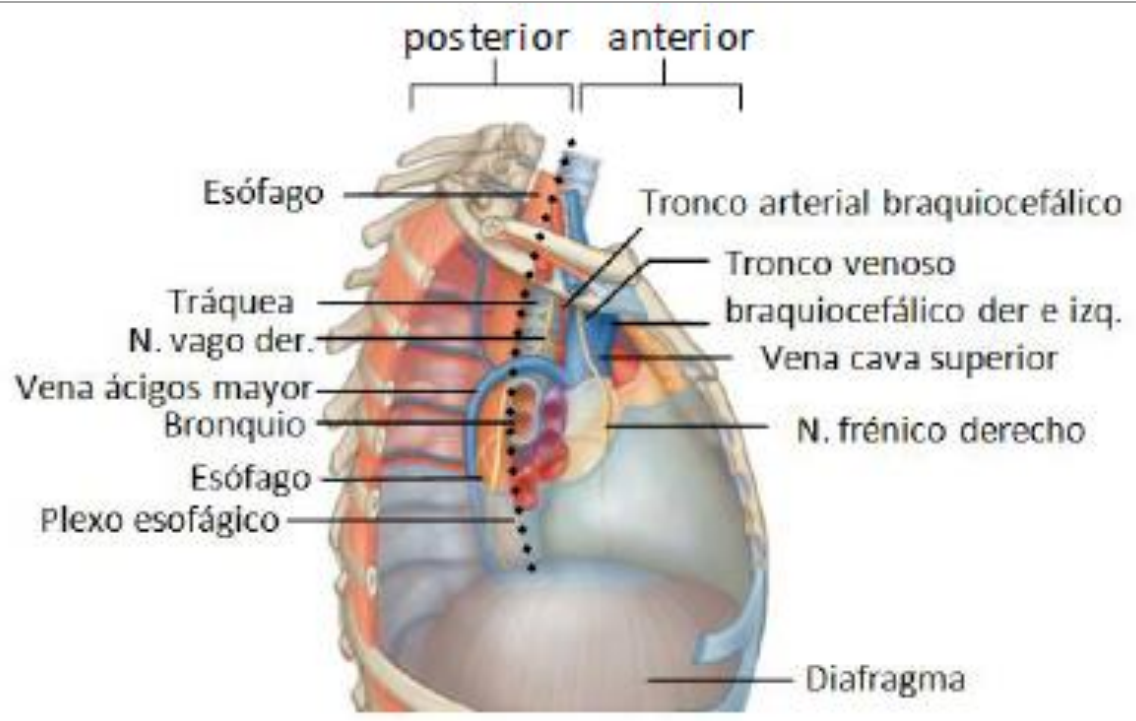

Figura 2. Vista lateral derecha del mediastino

Fuente: (Tramontini, 2019)

El mediastino contiene órganos que pueden sufrir tumores de carácter benigno o maligno por lo que esta región le procede a realizar tratamientos quirúrgicos. Los timomas, tumores neurogénicos y los quistes benignos justifican el 60\% de la patología quirúrgica del mediastino; los linfomas, teratomas y las enfermedades granulomatosas representan el 30\%, y el $10 \%$ restante corresponde a lesiones vasculares(Patané, y otros, 2006).Esta división en compartimientos es útil dado que las lesiones tumorales mediastínicas pueden facilitar o ayudar al diagnóstico según el compartimiento que ocupen(Ríos Rodríguez, Capin Sarria, \& Torres Aja, 2008).Ahora, las masas mediastinales del compartimento anterior son las más frecuentes y representan el 50\% de los tumores mediastinales(Torres-Rodríguez, y otros, 2016). El mediastino anterior se muestra en la Figura 3. 


\section{Síndrome mediastinal causas, diagnóstico y tratamiento}

Vol. 3, núm. 1., (2019)

Andrea Enriqueta Naranjo Castillo; Shyrle Lisbeth Vaca Rodríguez; María Gabriela Lucin Salazar; Danilo José Cabrera Galecio

De aquí se puede derivar la patología que pueden determinar las diversas manifestaciones clínicas que disminuyen la calidad de vida de los pacientes. Las enfermedades del mediastino tienen $\mathrm{u}$ origen sistémico, regional de vecindad, congénitas o adquiridas, primarias $\mathrm{o}$ metastásicas de órganos distantes (Euwing, 1989). Existen tres grupos de patología las cuales son los procesos inflamatorios, tumorales (quistes y tumores) y neoplasias mediastinales.

Dentro de los procesos inflamatorios encontramos las mediastinitis. Las mediastinitis se clasifican en agudas, necrotizantes y crónicas. Las primeras son debidas a perforación esofágica, tras exploraciones instrumentales o ingestión de cuerpos extraños, la segunda por procesos infecciosos o faringo-amigdalinos y la tercera por infecciones granulomatosas la cual determinan síndromes compresivos como el de la vena cava superior (Heras Gómez, 2006). 


\section{Síndrome mediastinal causas, diagnóstico y tratamiento}

Vol. 3, núm. 1., (2019)

Andrea Enriqueta Naranjo Castillo; Shyrle Lisbeth Vaca Rodríguez; María Gabriela Lucin Salazar; Danilo José Cabrera Galecio

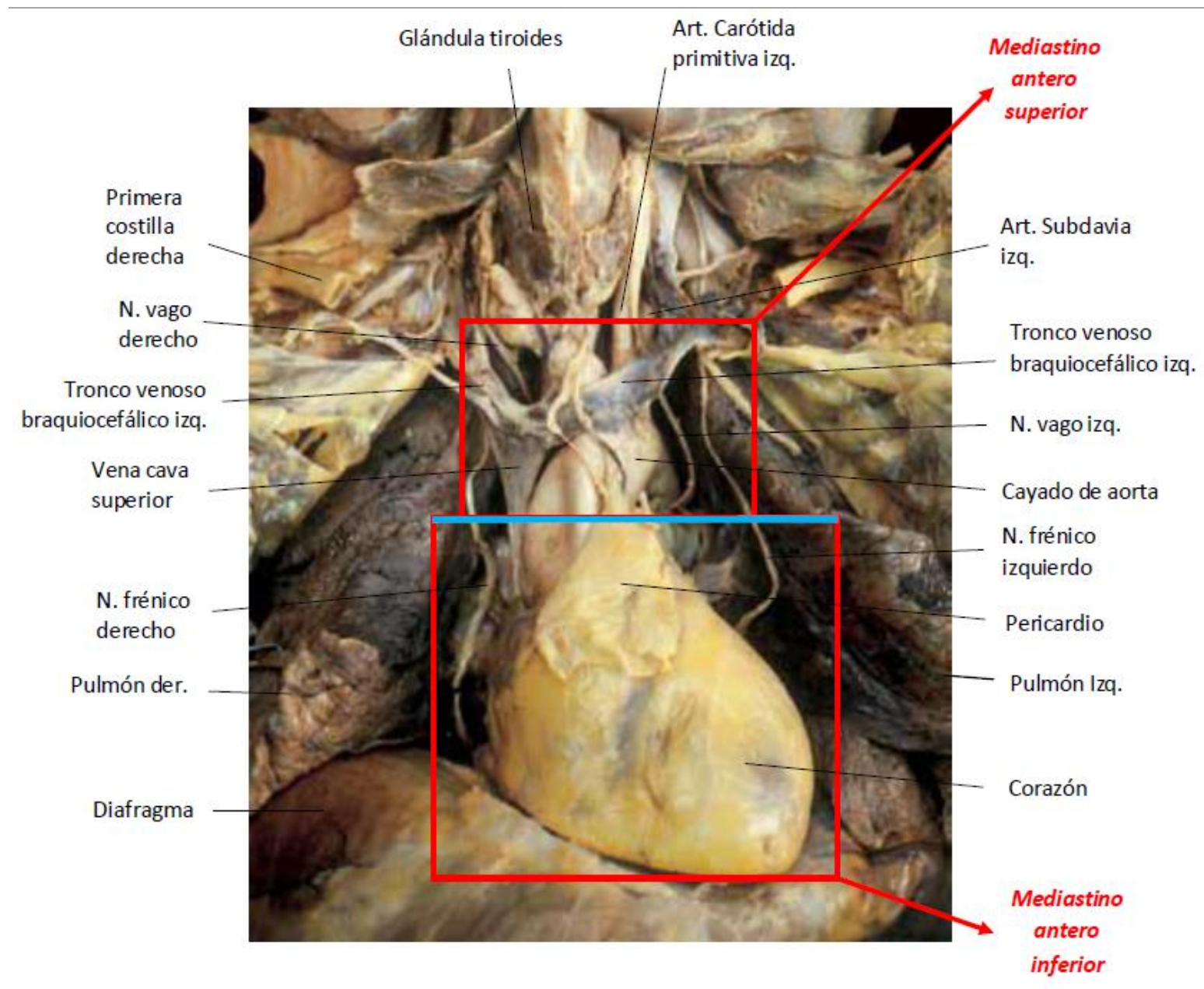

Figura 3. Vista del mediastino anterior

Fuente: (Tramontini, 2019)

Los tumores pueden formarse en cualquier parte del mediastino como el timo, en cordones nerviosos y en los linfáticos. En los adultos, el tumor se encuentra generalmente en la parte anterior (timoma maligno o linfoma), en cambio en los niños el tumor se suele ubicar en la parte posterior, se forma en los nervios, y por lo general es benigno(Rodriguez, y otros, 2013). En la Figura 4 se puede observar radiografía de un paciente de 41 años que presenta timoma. En 


\section{Síndrome mediastinal causas, diagnóstico y tratamiento}

Vol. 3, núm. 1., (2019)

Andrea Enriqueta Naranjo Castillo; Shyrle Lisbeth Vaca Rodríguez; María Gabriela Lucin Salazar; Danilo José Cabrera Galecio

(A) se detalla imagen densa que sobresale del borde inferior derecho del mediastino y en (B) control de radiología normal después de 4 años de extirpación quirúrgica.

(A)

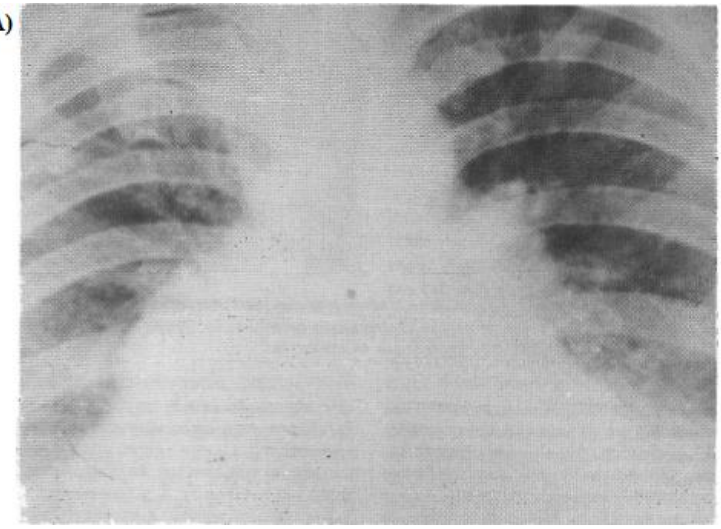

(B)

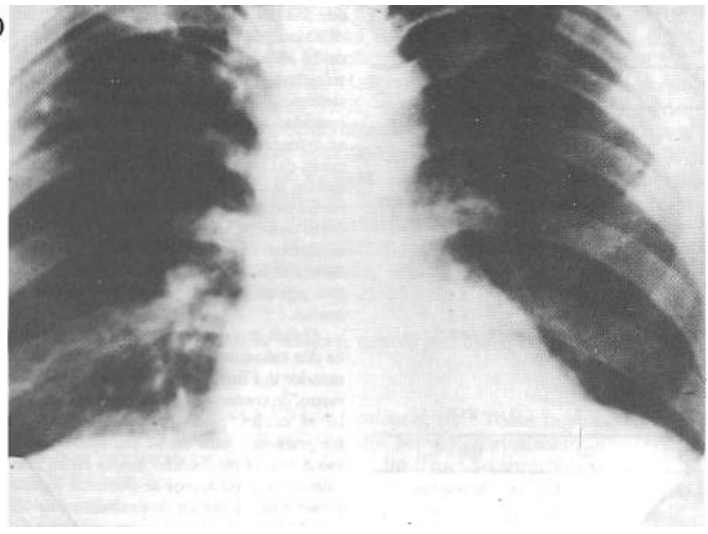

Figura 4. Radiografía de un paciente de 41 años que presenta timoma. En (A) se detalla imagen densa que sobresale del borde inferior derecho del mediastino y en (B) control de radiología normal después de 4 años de extirpación quirúrgica

Fuente: (Mendoza, 1989)

En cuanto a los quistes son lesionea benignas las cuales se ubican en el pericardio, la pleura, los bronquios o el esófago. Los quistes pleuropericárdicos se ubican en el mediastino anterior, los quistes broncogénicos se localizan en el mediastino medio y los quistes paraesofágicos en el mediastino posterior (Heras Gómez, 2006).

Las neoplasias mediastinales son poco frecuentes, pero pueden aparecer después de los 30 años o en avanzada edad. Aproximadamente $40 \%$ de las masas son asintomáticas y se descubren incidentalmente en una radiografía de tórax de rutina y el restante $60 \%$ tienen 


\section{Síndrome mediastinal causas, diagnóstico y tratamiento}

Vol. 3, núm. 1., (2019)

Andrea Enriqueta Naranjo Castillo; Shyrle Lisbeth Vaca Rodríguez; María Gabriela Lucin Salazar; Danilo José Cabrera Galecio

síntomas relacionados con la compresión o la invasión directa de las estructuras que rodean el mediastino, o a síndromes paraneoplásicos(Heras Gómez, 2006).

Poder determinar los síntomas de padecer tumores benignos y malignos se puede detallar en la Tabla 1. Los síntomas más frecuentes en los tumores malignos fueron dolor toráxico, pérdida de peso y datos secundarios a compresión de la vena cava superior; en los benignos los más frecuentes fueron disnea y tos(Salazar, Soto, Pucci, \& Brenes, 1980).

Para poder determinar el tipo de patología y así encontrar el tratamiento adecuado para el bienestar del paciente es necesario aplicar una serie de técnicas que permitan un buen diagnóstico. Estas técnicas son menos invasivas y tratar de técnicas inmunohistoquímicas y hasta de microscopia electrónica. Estas pueden ser ecocardiograma, TAC, biopsia percutánea guiada por TAC, procedimientos quirúrgicos, mediastinoscopia, mediastinotomía paraesternal anterior (procedimiento de Chamberlain) y la toracoscopía(Rodriguez, y otros, 2013). En la Figura 5 se detalla un ecocardiograma antes y después del tratamiento y en la Figura 6 se detalla una tomografía computarizada de tórax con contraste antes y después del tratamiento. 


\section{Síndrome mediastinal causas, diagnóstico y tratamiento}

Vol. 3, núm. 1., (2019)

Andrea Enriqueta Naranjo Castillo; Shyrle Lisbeth Vaca Rodríguez; María Gabriela Lucin Salazar; Danilo José Cabrera Galecio

Tabla 1. Síntomas de los tumores mediastino

\begin{tabular}{lrr}
\hline \hline & Malignos(13) & Benignos (25) \\
& & \\
Dolor toríxico & $62 \%$ & $12 \%$ \\
Pérdida de peso & $38 \%$ & $4 \%$ \\
Datos sugerentes de Síndrome de V.C. superior & $31 \%$ & -- \\
Dolor miembro superior & $15 \%$ & -- \\
Disnea & $23 \%$ & $24 \%$ \\
Tos & $15 \%$ & $80 \%$ \\
Fiebre & $15 \%$ & -- \\
Disfonía & $7.5 \%$ & $40 \%$ \\
Asintomáticos & $9 \%$ & $12 \%$ \\
Síntomas miasténicos & & \\
& & \\
\hline \hline
\end{tabular}

Fuente: (Salazar, Soto, Pucci, \& Brenes, 1980)

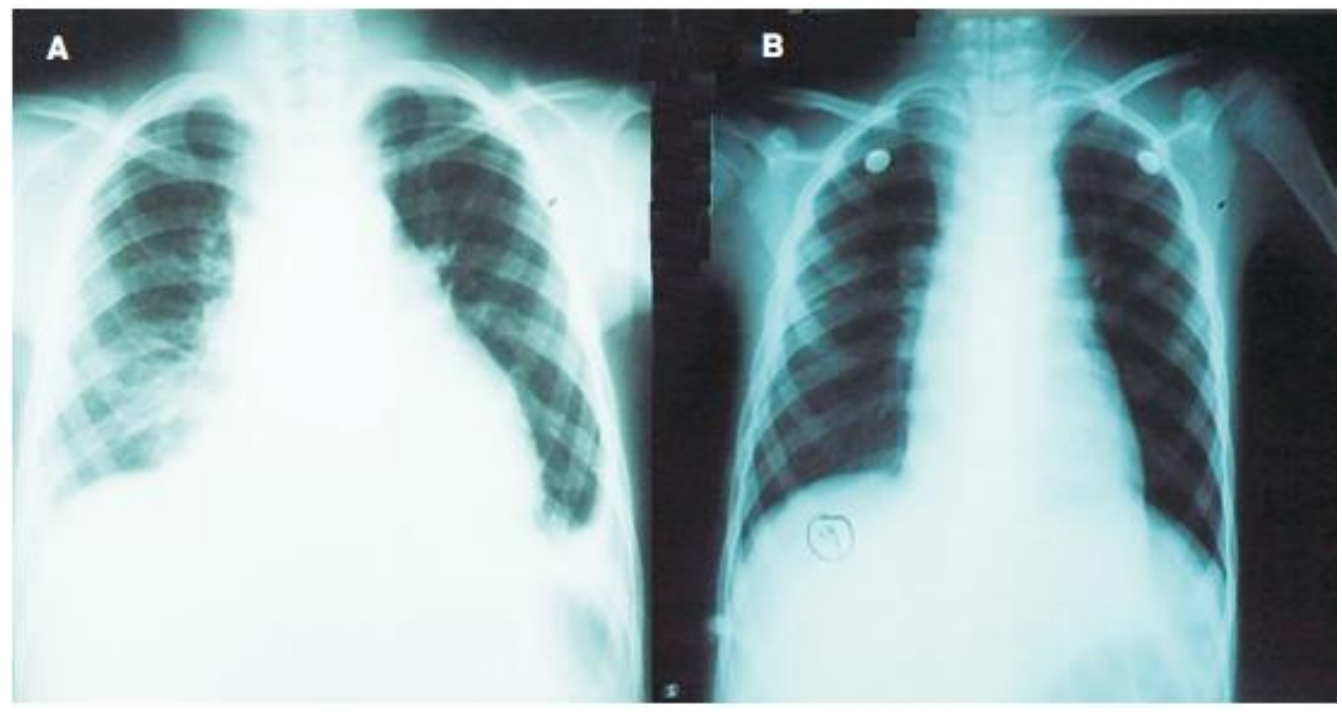

Figura 5. Radiografía de tórax en proyección postero-anterior. (A) Nótese el ensanchamiento del mediastino con aumento del índice cardiotorácico y obliteración de 


\section{Síndrome mediastinal causas, diagnóstico y tratamiento}

Vol. 3, núm. 1., (2019)

Andrea Enriqueta Naranjo Castillo; Shyrle Lisbeth Vaca Rodríguez; María Gabriela Lucin Salazar; Danilo José Cabrera Galecio

los ángulos costofrénicos bilaterales. (B) A las 72 horas de iniciada la terapia, la radiografía posteroanterior de tórax es reportada como normal

Fuente: (De los Reyes, y otros, 2010)

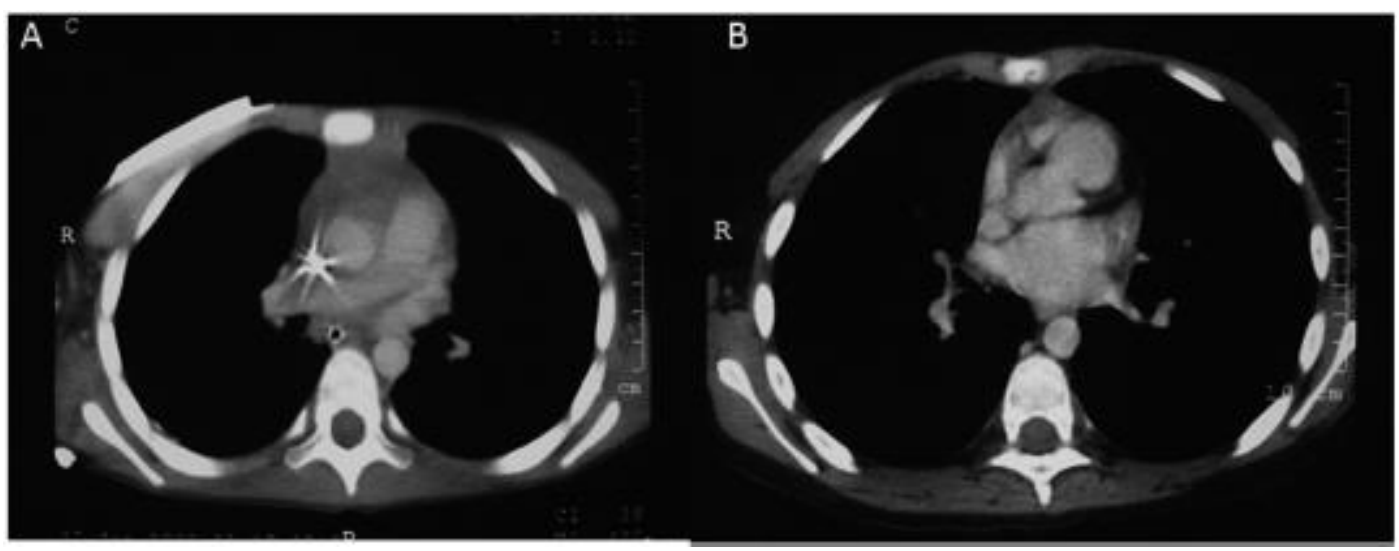

Figura 6. Tomografía computadorizada de tórax con contraste. A) Ventana para mediastino: se observa una masa en mediastino anterior, de densidad heterogénea con algunas áreas hipodensas por posible necrosis. No se evidencia obliteración de la vena cava superior. B) Tomografía computadorizada de tórax con contraste a las 72 horas de la terapia: nótese la disminución importante del tamaño de la masa de mediastino. El contorno del mediastino es normal para la edad

Fuente: (De los Reyes, y otros, 2010)

Después del diagnóstico realizado se plantea el tratamiento la cual implica el uso de anestésico. El uso de ciertos elementos para la anestesia puede facilitar la obstrucción de la tráquea o de los bronquios principales y el desarrollo de hipoxemia grave por compresión de los 


\section{Síndrome mediastinal causas, diagnóstico y tratamiento}

Vol. 3, núm. 1., (2019)

Andrea Enriqueta Naranjo Castillo; Shyrle Lisbeth Vaca Rodríguez; María Gabriela Lucin Salazar; Danilo José Cabrera Galecio

grandes vasos, la anticipación de estas complicaciones puede facilitar el tratamiento(De los Reyes, y otros, 2010).

Existen recomendaciones al aplicar tratamiento y en algunos casos tener algunas consideraciones con el anestésico a los pacientes. Estas son: mantener la respiración espontánea hasta donde sea posible, inducir y mantener la anestesia en posición sentado, obtener accesos vasculares en los miembros inferiores y, en algunos casos, podría ser necesario la instalación urgente de un bypass cardiopulmonar(De los Reyes, y otros, 2010).

Dentro de todos estos cuadros que forman parte de las urgencias cardiotorácicas, que originan un efecto compresivo de la masa, están el síndrome de la vena cava superior, el derrame pleural, el derrame pericárdico, el taponamiento cardiaco y el síndrome mediastinal (De los Reyes, y otros, 2010).

El síndrome mediastinal tiene mucha relevancia por el efecto que pueda producir estos tumores dentro del mediastino.

El síndrome mediastinal (SM) es un conjunto de síntomas y signos resultantes de la compresión de uno o varios órganos del mediastino, debida al aumento de tamaño (tumoral, inflamatorio o de otra naturaleza) de los órganos que lo constituyen, o por la compresión generada por el crecimiento de las estructuras aledañas. (Vega Lorenzo, y otros, 2016, pág. 62) 


\section{Síndrome mediastinal causas, diagnóstico y tratamiento}

Vol. 3, núm. 1., (2019)

Andrea Enriqueta Naranjo Castillo; Shyrle Lisbeth Vaca Rodríguez; María Gabriela Lucin Salazar; Danilo José Cabrera Galecio

Es decir, los quistes y tumores pueden también comprimir o infiltrar las estructuras adyacentes, dando lugar a síndromes diversos: vasculares, traqueobronquiales, digestivos, neurológicos, entre otros (Heras Gómez, 2006). Esto se puede observar en la Tabla 2.

Tabla 2. Síndromes mediastínicos debido a compresión o infiltración de las estructuras adyacentes.

\begin{tabular}{|c|c|}
\hline \multicolumn{2}{|c|}{ Síndromes mediastínicos } \\
\hline Clínicos & $\begin{array}{l}\text { Vasculares } \\
\text { - Arteriales. Venosos. Linfáticos (conducto torácico) } \\
\text { Cardiacos } \\
\text { - Alteraciones del ritmo. Angor. Taponamiento } \\
\text { Traqueobronquiales } \\
\text { - Tos. Disnea. Estridor } \\
\text { Digestivos } \\
\text { - Disfagia } \\
\text { Neurológicos } \\
\text { - Compresión medular. Disfonía. Afectación nervios } \\
\text { periféricos. Afectación simpática } \\
\text { Paraneoplásicos } \\
\text { - Miastenia. Alteraciones hematológicas }\end{array}$ \\
\hline Topográficos & $\begin{array}{l}\text { Anterosuperior } \\
\text { - Vena cava superior } \\
\circ \quad \text { Edema de cara, cuello y en esclavina. Cefalea. } \\
\text { Cianosis. Hinchazón párpados } \\
\text { - Compresión traqueal } \\
\circ \quad \text { Dificultad respiratoria } \\
\text { Anteroinferior } \\
\text { - Afectación cardiaca } \\
\circ \quad \text { Arritmias. Angor. Taponamiento } \\
\text { - Vena cava inferior } \\
\circ \quad \text { Hepatomegalia } \\
\text { - Afectación pleural } \\
\circ \quad \text { Derrame pleural } \\
\text { Medio } \quad \text { Traqueobronquial } \\
\text { - Tos. Disnea. Estridor } \\
\text { - Neurológico } \\
\circ \text { Frénico. Vago. Recurrente izquierdo } \\
\text { Posterior } \quad \text { Esofágico } \\
\circ \quad \text { Disfagia } \\
\text { - Osteomuscular } \\
\circ \quad \text { Dolores intercostales, pararaquídeos, etc. } \\
\text { - Nervioso } \\
\circ \quad \text { Compresión medular } \\
\text { - Sistema simpático } \\
\circ \text { Horner }\end{array}$ \\
\hline
\end{tabular}

Fuente: (Heras Gómez, 2006) 


\section{Síndrome mediastinal causas, diagnóstico y tratamiento}

Vol. 3, núm. 1., (2019)

Andrea Enriqueta Naranjo Castillo; Shyrle Lisbeth Vaca Rodríguez; María Gabriela Lucin Salazar; Danilo José Cabrera Galecio

Por lo tanto, esta investigación tiene como objetivo general analizar las causas, diagnósticos y tratamiento del síndrome mediastinal. La metodología empleada utiliza una investigación de tipo documental y bibliográfica.

\section{Método.}

Poder realizar el análisis a los efectos de la compresión de las masas o tumores dentro del mediastino requiere de una investigación de carácter documental y bibliográfico que permita poder encontrar las causas, diagnóstico y tratamiento de las patologías más frecuentes en el síndrome mediastinal. Para ello se contó con la ayuda de textos, documentos y artículos científicos publicados disponibles en la web.

\section{Resultados.}

\section{Clasificación del síndrome mediastinal}

Esta clasificación está determinada por el síndrome mediastinal superior y del síndrome mediastinal inferior.

Síndrome mediastinal superior.

Este síndrome está representado principalmente por el síndrome de vena cava superior (SVCS). El SVCS está caracterizada por la aparición en forma simultánea de una enfermedad vascular y otra benigna o maligna(Arribalzaga, Aguirre, \& Corchuelo, 2014).El 95\% de los casos está relacionado con procesos neoplásicos, siendo el carcinoma pulmonar el principal causante 


\section{Síndrome mediastinal causas, diagnóstico y tratamiento}

Vol. 3, núm. 1., (2019)

Andrea Enriqueta Naranjo Castillo; Shyrle Lisbeth Vaca Rodríguez; María Gabriela Lucin Salazar; Danilo José Cabrera Galecio

(85\%) y dentro de este el carcinoma pulmonar microcítico(Mateos Caballero, Márquez Pérez, Antona Rodríguez, \& Fuentes Otero, 2019).

La compresión vascular, principalmente venosa, se produce en un espacio relativamente reducido por lo que produce los síntomas como tos, disfonía, disnea y/o estridor y disfagia, cefalea, somnolencia, zumbidos de oídos debido al edema cerebral y aumento del diámetro del cuello(Arribalzaga, Aguirre, \& Corchuelo, 2014). Esto se puede observar en la Tabla 3.

\section{Tabla 3. Signos y síntomas del SVCS}

\section{Tabla II. Signos y sintomas del SVCS.}

\section{Signos}

Edema facial, cuello y hemitorax superior.

(edema en esclavina)

Cianosis facial

Pletora facial

Taquipnea

Telangiectasias en cuello y torax

( $2^{\mathrm{a}}$ a circulación colateral)
Sintomas

Disnea. Sintomas mas frecuente

Sensacion de embotamiento craneal

Tos

Cefalea

Dolor toracico que empeora al inclinarse hacia delate

Fuente: (Mateos Caballero, Márquez Pérez, Antona Rodríguez, \& Fuentes Otero, 2019)

Las causas pueden ser benignas o malignas según sea el caso del paciente tratante. Las primeras se caracterizan por procesos inflamatorios agudos, aneurismas del cayado aórtico, bocios tiroideos sumergidos y las segundas por cáncer pulmonar, linfoma, adenopatías metastásicas, timomas, enfermedades hematológicas (Arribalzaga, Aguirre, \& Corchuelo, 2014). Esta se muestra en la Tabla 4. 


\section{Síndrome mediastinal causas, diagnóstico y tratamiento}

Vol. 3, núm. 1., (2019)

Andrea Enriqueta Naranjo Castillo; Shyrle Lisbeth Vaca Rodríguez; María Gabriela Lucin Salazar; Danilo José Cabrera Galecio

\section{Tabla 4. Causas del SVCS}

\section{Tabla I. Causas de SVCS.}

Causas no tumorales

Fibrosis mediastinica idiopatica

Granulomas mediastinico por histoplasmosis

Mediastinitis tuberculosa

Trombosis por cateter o marcapasos
Causas tumorales

Cancer de pulmon (oat cell)

Linfoma mediastinico

Metastasis ganglionares mediastinicas (Ca. Mama,

melanoma, gastrointestinales)

Fuente: (Mateos Caballero, Márquez Pérez, Antona Rodríguez, \& Fuentes Otero, 2019)

El diagnostico utiliza técnica como RX tórax, TAC tórax y técnicas invasivas que permiten determinar el diagnostico histológico. Según Mateos Caballero, Márquez Pérez, Antona Rodríguez y Fuentes Otero (2019)estas técnicas son:

$\checkmark$ Citología de esputo: puede ser muy rentable ya que hemos comentado que la principal causa de este síndrome es el carcinoma pulmonar microcítico.

$\checkmark \quad$ Broncofibroscopia, por igual motivo.

$\checkmark$ Biopsia glanglionar de estaciones periféricas accesibles y palpables como cervicales, axilares o supraclaviculares.

$\checkmark \quad$ PAAF con control de TAC.

$\checkmark \quad$ Mediastinoscopia

$\checkmark \quad$ Toracotomía.(Pág. 230) 


\section{Síndrome mediastinal causas, diagnóstico y tratamiento}

Vol. 3, núm. 1., (2019)

Andrea Enriqueta Naranjo Castillo; Shyrle Lisbeth Vaca Rodríguez; María Gabriela Lucin Salazar; Danilo José Cabrera Galecio

El tratamiento puede depender del diagnóstico y la patología de la enfermedad. Pasa de realizar quimioterapia, radioterapia o de medidas generales. Estas últimas están representadas por:

$\checkmark$ Oxigenoterapia y reposo en cama con el cabecero incorporado $\left(45^{\circ}\right)$.

$\checkmark$ Diuréticos y dieta pobre en sal: Furosemida $40 \mathrm{mg} / 24$ horas.

$\checkmark$ Esteroides: Puede mejorar la sintomatología al disminuir el componente inflamatorio del tumor. Dexametasona 6-8 mg cada 6 u 8 horas.(Mateos Caballero, Márquez Pérez, Antona Rodríguez, \& Fuentes Otero, 2019, pág. 230)

La expectativa de vida, dependiendo de la patología donde hay presencia de malignidad es de 6 meses. En algunos pacientes el tratamiento de resección y colocación de prótesis vascular con radioterapia posterior llevó a una supervivencia de 4 años(Arribalzaga, Aguirre, \& Corchuelo, 2014).

\section{Síndrome mediastinal medio}

Los signos y síntomas de la compresión de la tráquea, bronquios, del recurrente izquierdo, del nervio frénico, del tronco braquiocefálico o de la subclavia y del nervio vago se muestran en la Tabla 5. 


\section{Síndrome mediastinal causas, diagnóstico y tratamiento}

Vol. 3, núm. 1., (2019)

Andrea Enriqueta Naranjo Castillo; Shyrle Lisbeth Vaca Rodríguez; María Gabriela Lucin Salazar; Danilo José Cabrera Galecio

Tabla 5. Signos y síntomas que provienen del síndrome mediastinal medio

\begin{tabular}{ll}
\hline Órganos del Mediastino Medio & \multicolumn{1}{c}{ Signos y Síntomas } \\
\hline Tráquea & Disnea inspiratoria, tiraje, tos ronca y cianosis \\
\hline Bronquios & $\begin{array}{l}\text { Aparece tiraje, murmullo vesicular disminuido o } \\
\text { abolido, disminución de la expansión y en el caso de } \\
\text { oclusión total, atelectasia del lado afectado }\end{array}$ \\
\hline Recurrente izquierdo & $\begin{array}{l}\text { Parálisis de la cuerda vocal izquierda acompañada de } \\
\text { tos y voz bitonal. }\end{array}$ \\
\hline Nervio frénico & $\begin{array}{l}\text { hemiparesia del diafragma y, a veces hipo y dolores } \\
\text { neurálgicos }\end{array}$ \\
\hline Tronco braquiocefálico o de la & $\begin{array}{l}\text { Produce frémito, soplo y pulsos radiales diferentes } \\
\text { subclavia }\end{array}$ \\
\hline & $\begin{array}{l}\text { Tos coqueluchoide, } \\
\text { intermitentes, disfagia intermitente y bradicardia en } \\
\text { caso de irritación. También puede producir vómitos y } \\
\text { gastralgia }\end{array}$ \\
\hline
\end{tabular}

Fuente: (Mateos Caballero, Márquez Pérez, Antona Rodríguez, \& Fuentes Otero, 2019)

Ahora, se puede determinar las enfermedades que forman parte del mediastino medio a través de la epidemiologia con su diagnóstico de cada una de ellas, como se detalla en la Tabla 6. 


\section{Síndrome mediastinal causas, diagnóstico y tratamiento}

Vol. 3, núm. 1., (2019)

Andrea Enriqueta Naranjo Castillo; Shyrle Lisbeth Vaca Rodríguez; María Gabriela Lucin Salazar; Danilo José Cabrera Galecio

Tabla 6. Epidemiologia y diagnóstico de los síndromes del mediastino medio

\begin{tabular}{|c|c|c|}
\hline $\begin{array}{l}\text { Síndrome del Mediastino } \\
\text { Medio }\end{array}$ & Epidemiologia & Diagnóstico \\
\hline $\begin{array}{l}\text { QUISTE } \\
\text { PLEUROPERICÁRDICO }\end{array}$ & $\begin{array}{l}\text { Masa pericárdica primaria más } \\
\text { frecuente. Es secundario al } \\
\text { atrapamiento de una porción } \\
\text { de pericardio durante el } \\
\text { desarrollo embrionario, que da } \\
\text { lugar a una masa con pared de } \\
\text { tejido conectivo formado por } \\
\text { una única capa de células } \\
\text { mesoteliales y cuyo contenido } \\
\text { es líquido y unilocular }\end{array}$ & $\begin{array}{l}\text { TCMD: Lesión hipodensa } \\
\text { de bordes redondeados y } \\
\text { bien definidos que no } \\
\text { realza con cte IV. }\end{array}$ \\
\hline $\begin{array}{l}\text { QUISTE } \\
\text { BRONCOGÉNICO }\end{array}$ & $\begin{array}{l}\text { Alteración en la segmentación } \\
\text { anormal del árbol } \\
\text { traqueobronquial durante el } \\
\text { desarrollo embrionario del } \\
\text { intestino anterior, dando lugar } \\
\text { a una masa delimitada por } \\
\text { epitelio columnar } \\
\text { pseudoestratificado, músculo } \\
\text { liso y tejido glandular, que se } \\
\text { rellena de líquido sero- } \\
\text { mucoso. }\end{array}$ & $\begin{array}{l}\text { TCMD: Lesión ovoidea, } \\
\text { generalmente de baja } \\
\text { atenuación, de bordes } \\
\text { bien definidos y pared } \\
\text { fina, sin realce de cte IV. } \\
\text { RM: T1: intensidad de } \\
\text { señal variable, desde baja } \\
\text { (similar al líquido), a alta } \\
\text { (contenido proteico) y T2: } \\
\text { hiperintensidad de señal. }\end{array}$ \\
\hline $\begin{array}{l}\text { QUISTE } \\
\text { DUPLICACIÓN } \\
\text { ESOFÁGICA }\end{array}$ & $\begin{array}{l}\text { Anomalía en la segmentación } \\
\text { posterior del intestino anterior. } \\
\text { Habitualmente contienen } \\
\text { mucosa gástrica o pancreática; } \\
\text { ello conlleva un mayor riesgo } \\
\text { de infección, perforación y } \\
\text { hemorragia }\end{array}$ & $\begin{array}{l}\text { TCMD: masa homogénea } \\
\text { de morfología tubular, } \\
\text { bordes regulares y bien } \\
\text { definidos. } \\
\text { RM: T1: intensidad de } \\
\text { señal variable, desde baja } \\
\text { (similar al líquido), a alta } \\
\text { (contenido proteico) y T2: } \\
\text { hiperintensidad de señal. } \\
\text { Gammagrafía }\end{array}$ \\
\hline
\end{tabular}


Síndrome mediastinal causas, diagnóstico y tratamiento

Vol. 3, núm. 1., (2019)

Andrea Enriqueta Naranjo Castillo; Shyrle Lisbeth Vaca Rodríguez; María Gabriela Lucin Salazar; Danilo José Cabrera Galecio

\begin{tabular}{|c|c|c|}
\hline & & $\begin{array}{l}\text { pernectato Tc } 99 \mathrm{~m} \text { puede } \\
\text { ayudar a identificar } \\
\text { quistes con mucosa } \\
\text { gástrica ectópica que } \\
\text { puede mostrar signos de } \\
\text { sangrado }\end{array}$ \\
\hline $\begin{array}{l}\text { PÓLIPO GIGANTE } \\
\text { FIBROVASCULAR } \\
\text { ESOFÁGICO }\end{array}$ & $\begin{array}{l}\text { Los pólipos gigantes } \\
\text { fibrovasculares son tumores } \\
\text { raros benignos intraluminales } \\
\text { esofágicos. Son de crecimiento } \\
\text { lento, pedunculados que } \\
\text { normalmente se originan desde } \\
\text { la pared del tercio superior del } \\
\text { esófago. Puede alcanzar } \\
\text { grandes dimensiones }\end{array}$ & $\begin{array}{l}\text { TC y RM especialmente } \\
\text { con las reconstrucciones } \\
\text { multiplanares se puede } \\
\text { obtener información que } \\
\text { indique su localización } \\
\text { intraluminal en el esófago } \\
\text { y que es pedunculado }\end{array}$ \\
\hline LINFOMA & $\begin{array}{l}\text { Los pacientes con linfoma } \\
\text { mediastínico primario suelen } \\
\text { tener una masa mediastínica } \\
\text { anterior a menudo asociada } \\
\text { con adenopatías en los } \\
\text { compartimentos medio y } \\
\text { posterior. Menos } \\
\text { frecuentemente, el linfoma } \\
\text { puede aparecer como una masa } \\
\text { homogénea paravertebral, } \\
\text { difícil de distinguir de otros } \\
\text { tumores como } \\
\text { neurogénicos. }\end{array}$ & $\begin{array}{l}\text { TCMD: adenopatías o } \\
\text { conglomerados } \\
\text { adenopáticos que pueden } \\
\text { mostrar cambios quísticos } \\
\text { o necróticos. } \\
\text { 18F-FDG PET-TC: se } \\
\text { recomienda realizarlo 6-8 } \\
\text { semanas después del } \\
\text { tratamiento en pacientes } \\
\text { con linfoma de Hodgkin y } \\
\text { en el linfoma difuso de } \\
\text { células B }\end{array}$ \\
\hline $\begin{array}{l}\text { ADENOPATÍAS } \\
\text { TUMORALES }\end{array}$ & $\begin{array}{l}\text { Ganglios linfáticos del } \\
\text { mediastino medio y posterior } \\
\text { también pueden estar } \\
\text { implicados en los procesos } \\
\text { inflamatorios/granulomatosos, } \\
\text { como la sarcoidosis, } \\
\text { enfermedad de Castleman, } \\
\text { neumoconiosis, así como en } \\
\text { las infecciones, la mayoría } \\
\text { VIH y Mycobacterium }\end{array}$ & $\begin{array}{l}\text { las imágenes no son } \\
\text { específicas y deben ser } \\
\text { evaluadas con el resto de } \\
\text { pruebas del estudio } \\
\text { diagnóstico. }\end{array}$ \\
\hline
\end{tabular}


Síndrome mediastinal causas, diagnóstico y tratamiento

Vol. 3, núm. 1., (2019)

Andrea Enriqueta Naranjo Castillo; Shyrle Lisbeth Vaca Rodríguez; María Gabriela Lucin Salazar; Danilo José Cabrera Galecio

\begin{tabular}{|c|c|c|c|}
\hline & & tuberculosis. & \\
\hline LIPOBLASTOMA & & $\begin{array}{l}\text { Tumor benigno raro casi } \\
\text { exclusivo de la edad pediátrica. } \\
\text { Está formado por tejido } \\
\text { adiposo fetal que contiene } \\
\text { adipocitos con distintos grados } \\
\text { de maduración: } \\
\text { Lipocitosunivacuolados, } \\
\text { lipoblastosmultivacuolados }\end{array}$ & $\begin{array}{l}\text { TCMD: Distinta } \\
\text { proporción de grasa y } \\
\text { partes blandas. Más grasa } \\
\text { en pacientes más jóvenes. } \\
\text { Su localización más } \\
\text { frecuente son los } \\
\text { miembros y el cuello }\end{array}$ \\
\hline $\begin{array}{l}\text { LIPOMA } \\
\text { LIPOSARCOMA }\end{array}$ & $\mathbf{Y}$ & $\begin{array}{l}\text { Son tumores de estirpe grasa } \\
\text { de lento y asintomático } \\
\text { crecimiento, por lo que } \\
\text { usualmente presentan un gran } \\
\text { tamaño al diagnóstico. }\end{array}$ & $\begin{array}{l}\text { TCMD: } \\
\text { Lipoma: masa homogénea } \\
\text { de atenuación grasa, } \\
\text { homogénea y bien } \\
\text { definida, que no invade } \\
\text { estructuras vecinas } \\
\text { Liposarcoma: masa } \\
\text { extensa y heterogénea que } \\
\text { contiene grasa, gruesos } \\
\text { septos fibrosos y tejido de } \\
\text { atenuación partes blandas } \\
\text { y que infiltran de manera } \\
\text { difusa las estructuras } \\
\text { mediastínicas. } \\
\text { RM: } \\
\text { Lipoma: hiperintensidad } \\
\text { de señal T1 y T2 y } \\
\text { supresión de la señal en } \\
\text { secuencias fat-sat. } \\
\text { Liposarcoma: masa de } \\
\text { señal heterogénea con } \\
\text { realce heterogéneo de } \\
\text { contraste. }\end{array}$ \\
\hline HERNIA HIATAL & & $\begin{array}{l}\text { Es la masa mediastínica más } \\
\text { frecuente. Se trata de la }\end{array}$ & $\begin{array}{l}\text { RX de tórax y TC: Masa } \\
\text { en el mediastino medio, a }\end{array}$ \\
\hline
\end{tabular}




\section{Síndrome mediastinal causas, diagnóstico y tratamiento}

Vol. 3, núm. 1., (2019)

Andrea Enriqueta Naranjo Castillo; Shyrle Lisbeth Vaca Rodríguez; María Gabriela Lucin Salazar; Danilo José Cabrera Galecio

\begin{tabular}{|c|c|c|}
\hline & $\begin{array}{l}\text { herniación de elementos de la } \\
\text { cavidad abdominal en el tórax. } \\
\text { Hay dos tipos principales de } \\
\text { hernias de hiato: deslizamiento } \\
\text { y paraesofágica. En una hernia } \\
\text { por deslizamiento, la unión } \\
\text { gastroesofágica migra por } \\
\text { encima del diafragma a través } \\
\text { del hiato esofágico; en una } \\
\text { hernia paraesofágica, el } \\
\text { estómago se hernia sin ningún } \\
\text { desplazamiento de la unión } \\
\text { gastroesofágica. Las hernias } \\
\text { por deslizamiento, que } \\
\text { representan más del 95\% de } \\
\text { los casos, a menudo están } \\
\text { asociadas con la enfermedad } \\
\text { por reflujo gastroesofágico. } \\
\text { Por el contrario, las hernias } \\
\text { paraesofágicas pueden } \\
\text { presentarse de forma aguda } \\
\text { con síntomas obstructivos } \\
\text { secundarios a un vólvulo } \\
\text { gástrico, que puede provocar } \\
\text { sangrado, incarceración, } \\
\text { estrangulación y/o perforación } \\
\text { del estómago }\end{array}$ & $\begin{array}{l}\text { menudo con nivel } \\
\text { hidroaéreo, sin realce de } \\
\text { cte IV }\end{array}$ \\
\hline $\begin{array}{l}\text { HERNIA } \\
\text { BOCHDALEK }\end{array}$ & $\begin{array}{l}\text { En la hernia de Bochdalek, la } \\
\text { grasa abdominal (y en } \\
\text { ocasiones } \\
\text { determinadas vísceras) se } \\
\text { introducen en el tórax a través } \\
\text { de un defecto en la } \\
\text { posteromedial del diafragma } \\
\text { de predominio en el lado } \\
\text { izquierdo. La mayoría de las } \\
\text { hernias más grandes y } \\
\text { sintomáticas } \\
\text { diagnosticados durante el } \\
\text { período neonatal, debido a que } \\
\text { la hernia de órganos } \\
\text { abdominales } \\
\text { dificultad respiratoria grave. }\end{array}$ & $\begin{array}{l}\text { TC y RM: permiten } \\
\text { identificar la } \\
\text { discontinuidad de la } \\
\text { musculatura } \\
\text { diafragmática, así como } \\
\text { una masa homogénea de } \\
\text { atenuación grasa que se } \\
\text { introduce en la caja } \\
\text { torácica. Además de } \\
\text { grasa, pueden pasar } \\
\text { órganos abdominales a } \\
\text { través del defecto } \\
\text { diafragmático como el } \\
\text { intestino, el bazo, hígado, } \\
\text { estómago, riñón y } \\
\text { páncreas en las hernias de }\end{array}$ \\
\hline
\end{tabular}




\section{Síndrome mediastinal causas, diagnóstico y tratamiento}

Vol. 3, núm. 1., (2019)

Andrea Enriqueta Naranjo Castillo; Shyrle Lisbeth Vaca Rodríguez; María Gabriela Lucin Salazar; Danilo José Cabrera Galecio

\begin{tabular}{|l|l|l|}
\hline & Sin embargo, la hernia de & Bochdalek. Las \\
Bochdalek asintomática puede & reconstrucciones MPR del \\
encontrarse casualmente en el & TC son muy útiles para \\
$10,5 \%$ de los adultos & mostrar el defecto \\
sometidos estudios de imagen & diafragmático y los \\
torácicos. & & contenidos del saco \\
herniario.
\end{tabular}

Fuente: (Díaz, y otros, 2018)

De los anteriores síndromes del mediastino medio uno de los más importantes son los linfomas. La frecuencia mundial aproximada de linfomas de Hodgkin (LH) y no Hodgkin (LNH) es de 2.7 a 14.8\%; es la segunda neoplasia más frecuente en niños después de las leucemias(Calderón Elvir, 2005).

El tratamiento para los linfomas depende de las biopsias que se realicen y que sea adecuada para el estudio histopatológico. Aun cuando los estudios de imagen ayudan a la localización, extensión y estadificación del tumor, la biopsia es indispensable para estudios de inmunohistoquímica, citogenética y biología molecular especializados (Calderón Elvir, 2005).

\section{Sindrome mediastinal inferior}

El síndrome más importante dentro de este grupo es el síndrome de la vena cava inferior. Las anomalías congénitas son resultado de la persistencia o regresión anormal de una de las venas embrionarias precursoras, donde mostraron ser factor de riesgo para desarrollar trombosis venosa profunda, principalmente en las venas ilíacas y en pacientes jóvenes(López, Quiroz Castro, \& Ramírez Arias, 2012). Es decir, que se produce compresión de la vena cava inferior dando lugar a hepatomegalia, ascitis, edemas en extremidades inferiores, esplenomegalia y 


\section{Síndrome mediastinal causas, diagnóstico y tratamiento}

Vol. 3, núm. 1., (2019)

Andrea Enriqueta Naranjo Castillo; Shyrle Lisbeth Vaca Rodríguez; María Gabriela Lucin Salazar; Danilo José Cabrera Galecio

circulación colateral en abdomen(Mateos Caballero, Márquez Pérez, Antona Rodríguez, \& Fuentes Otero, 2019).

El diagnostico de esta enfermedad es realizada por TCMD a través de su alta resolución espacial, capacidad de análisis multiplanar y la utilización de medio contraste intravascular. Las anomalías congénitas de la VCI generalmente son hallazgos incidentales en TCMD; sin embargo, tienen importantes implicaciones clínicas y quirúrgicas y pueden dar pie a confusiones en los estudios de imagen por lo que es necesario conocerlas(López, Quiroz Castro, \& Ramírez Arias, 2012).

Aplicar otras técnicas que impliquen imágenes puede acarrear errores de diagnóstico produciendo riesgo de efectos colaterales. Las anomalías de este síndrome tienen diferentes tipos de implicaciones clínicas y quirúrgicas que incluyen mayor riesgo de trombosis venosa profunda y/o insuficiencia venosa crónica, riesgo potencial de complicaciones en cirugías de abdomen o tórax(López, Quiroz Castro, \& Ramírez Arias, 2012).

\section{Síndrome mediastinal posterior}

Los tumores que puedan presentarse en este nivel son de origen neurogénico por lo que son frecuente en niños. De estos tumores los más frecuentes son el neuroblastoma y el neurofibroma.

El neuroblastoma es un tumor maligno de las células de la cresta neural que lo sufren pacientes menores a 5 años de edad(Díaz, y otros, 2018). Su presentación clínica es variable; se 


\section{Síndrome mediastinal causas, diagnóstico y tratamiento}

Vol. 3, núm. 1., (2019)

Andrea Enriqueta Naranjo Castillo; Shyrle Lisbeth Vaca Rodríguez; María Gabriela Lucin Salazar; Danilo José Cabrera Galecio

debe a la compresión de estructuras vecinas; pueden causar disfagia y dificultad respiratoria; compresión del ganglio estrellado, lo que produce el síndrome de Horner(Calderón Elvir, 2005).

Para el diagnostico de esta enfermedad es necesario aplicar las técnicas de TCMD y MIBG. El primero muestra masa heterogénea, no encapsulada, con áreas variables de hemorragia, necrosis y realce, y se localizan en cadena simpática; y el segundo es altamente sensible para determinar la extensión de la enfermedad en los neuroblastomas productores de catecolaminas (S88\% y E98\%)(Díaz, y otros, 2018).

El tratamiento es la resección completa. Cuando hay tumores inoperables es recomendable la aplicación de quimioterapia. Hay informes que aconsejan la resección total, incluso en los de alto riesgo (estadio IV o de más de un año de evolución al momento del diagnóstico), sobre todo después de la quimioterapia(Calderón Elvir, 2005).

El neurofribroma es el segundo tumor neurogénico más frecuente y se desarrollan en la adolescencia. Son tumores benignos debidos a la proliferación desorganizada de todos los componentes celulares del nervio. Son la manifestación de un desorden genético autosómico dominante como la neurofibromatosis tipo 1 (NFl) (Calderón Elvir, 2005).

Las características de estos tumores son plexiformes de formación congénita. Los signos se manifiestan afectando el cráneo, la cara, la región paraespinal, el mediastino y el retroperitoneo(Calderón Elvir, 2005). El diagnostico a realizar es a través de TCMD con una atenuación de partes blandas homogénea la cual pueden producir erosión/ deformidad de costillas y/o vertebras y expansión de forámenes neurales; y RX T2W heterogénea con zona central de baja señal(Díaz, y otros, 2018). Cuando producen compresión de la médula espinal o 


\section{Síndrome mediastinal causas, diagnóstico y tratamiento}

Vol. 3, núm. 1., (2019)

Andrea Enriqueta Naranjo Castillo; Shyrle Lisbeth Vaca Rodríguez; María Gabriela Lucin Salazar; Danilo José Cabrera Galecio

de las raíces nerviosas, el tratamiento de elección es la resección quirúrgica(Calderón Elvir, 2005).

\section{Discusión y conclusiones.}

Una del parte más importante que tiene el cuerpo humano es el tórax. Dentro de él se encuentra el mediastino donde coagulan una serie de órganos que tienen gran relevancia en la funcionabilidad del organismo. Todo esto genera un equilibrio estático y dinámico producto del espacio generado entre cada uno de los órganos, pero también cumple con la función de permitir la circulación venosa de los mismos y permitir el paso de estructuras digestivas. El mediastino también está rodeado de la columna vertebral y el esternón. Es por ello que al padecer alguna enfermedad podría acarrear efectos colaterales tanto en los órganos que representa como también en los órganos vecinos del mediastino.

Estas enfermedades pueden acarrear compresión en muchos de los órganos a través demalformaciones benignas o malignas, según sea el caso. Poder determinar la ubicación es importante ya que con ella se puede derivar las causa, el diagnóstico y el tratamiento a al cual se deba realizar el paciente. Es aquí donde el mediastino se clasifica en tres partes. Mediastino anterior, medio y posterior. Las masas tumorales del mediastino son más frecuentes en el anterior ya que representan un $50 \%$ de los tumores. Dentro de estas enfermedades se clasifican en procesos inflamatorios, quistes y tumores. La primera predomina la mediastinitis, en la segunda y tercera están diversos quistes y tumores que tienen origen congénito y adquirido o pueden ser metastásicos y aparecen en la parte anterior en los adultos y en la parte posterior los niños. Los quistes son de característica benigna pero los tumores tienden a ser neoplasias mediastinales. 


\section{Síndrome mediastinal causas, diagnóstico y tratamiento}

Vol. 3, núm. 1., (2019)

Andrea Enriqueta Naranjo Castillo; Shyrle Lisbeth Vaca Rodríguez; María Gabriela Lucin Salazar; Danilo José Cabrera Galecio

Una de las enfermedades más recurrentes que puede sufrir el mediastino es el síndrome mediastinal que no es más que la compresión que pueden sufrir los órganos que la conforman debido a la presión que ejercen las masas o tumores que se forman dentro de él. Esto produce síndromes diversos que afectan los sistemas vasculares digestivos, neurológicos, tráquea, bronquios, entre otros.

Para poder determinar el tipo y origen del síndrome mediastinal es necesario poder clasificarlo, dependiendo también de la ubicación en el mediastino. Esta clasificación corresponde a síndrome mediastinal superior, síndrome mediastinal inferior, síndrome mediastinal medio y el síndrome mediastinal posterior. Para el primero, el síndrome de la vena cava superior es el que más frecuencia se determina. Para el segundo, el que prevalece es el síndrome de la vena cava inferior. Para el tercero, el síndrome o enfermedad que prevalece es el linfoma y para el cuarto están los neuroblastomas y neurfibromas los síntomas generales son tos, disnea y dolor torácico. Las causas son diversas y dependen de cada una de las patologías. El hallazgo de estas enfermedades ocurre de manera incidental. Dependiendo de la histología del paciente se aplican radiografías, TCMD, resonancia magnética o gammagrafías como técnicas diagnósticas. El tratamiento predominante es extracción quirúrgica o aplicación de quimioterapia o radioterapia según sea el caso, lo cual va permitir una mejor calidad de vida del paciente y sus familiares.

\section{Referencias.}

Arribalzaga, E., Aguirre, M., \& Corchuelo, C. (2014). Conducta en el síndrome de vena cava superior (SVCS). Revista chilena de cirugía, 66(1), 71-77. 


\section{Síndrome mediastinal causas, diagnóstico y tratamiento}

Vol. 3, núm. 1., (2019)

Andrea Enriqueta Naranjo Castillo; Shyrle Lisbeth Vaca Rodríguez; María Gabriela Lucin

Salazar; Danilo José Cabrera Galecio

Calderón Elvir, C. A. (2005). Diagnóstico y tratamiento quirúrgico de masas mediastinales en niños. Acta Pediatr Mex; 26(2), 79-89.

De los Reyes, I., Saavedra, C., Quijano, S., Varón, A., Moreno, C. E., \& Jurado, M. (2010). Tumores malignos del mediastino en niños: un problema clínico urgente. Biomédica, vol. $30,27-31$

Díaz, A. M., Rodríguez, L. F., Peña, M. F., Fernández, I. P., Rodríguez, M. I., \& T., S. M. (2018). Tumores de mediastino medio y posterior. Seram. Sociedad española de radiologia médica, 1-34.

Euwing, C. M. (1989). Tumores y quistes primarios del mediastino: diagnóstico y tratamiento quirúrgico. In Anales de la Facultad de Medicina (Vol. 55, No. 1). Universidad Nacional Mayor de San Marcos., 55-60.

Heras Gómez, F. (2006). Patología mediastínica. Medicina Respiratoria, 2, 1229-1241.

Ibarra-Pérez, C., Kelly-García, J., \& Fernández-Corzo, M. A. (2001). Guía diagnósticoterapéutica: tumores y masas del mediastino. Revista del Instituto Nacional de Enfermedades Respiratorias, 14(3), 172-177.

López, A. E., Quiroz Castro, Ó., \& Ramírez Arias, J. L. (2012). Anomalías congénitas de la vena cava inferior. El rol de la tomografía computada multidetector. In Anales de Radiología, México (Vol. 11, No. 2)., 104-113.

Mateos Caballero, L., Márquez Pérez, F. L., Antona Rodríguez, M. J., \& Fuentes Otero, F. (27 de Abril de 2019). Manejo del paciente con patología mediastínica. Obtenido de https://www.neumosur.net/files/EB03-20\%20patologia\%20mediastinica.pdf

Mendoza, C. (1989). Tumores y quistes primarios del mediastino: Diagnóstico y tratamiento quirúrgico. An Fac Med UNMSM II. $N^{\circ}$ 1. Dsiponible en: http://scielo.iics.una.py/pdf/sopaci/v37n2/v37n2a06.pdf, 55-60. 


\section{Síndrome mediastinal causas, diagnóstico y tratamiento}

Vol. 3, núm. 1., (2019)

Andrea Enriqueta Naranjo Castillo; Shyrle Lisbeth Vaca Rodríguez; María Gabriela Lucin Salazar; Danilo José Cabrera Galecio

Patané, A. K., Poleri, C., Olmedo, G., Nieva, B., Rosenberg, M., Rojas, O., \& Abdala, O. (2006). Tumores Primarios de Mediastino. Revista Americana de Medicina Respiratoria, 6(1), 47-50.

Ríos Rodríguez, A., Capin Sarria, N., \& Torres Aja, L. (2008). Tumores de mediastino: informe sobre 29 pacientes. Revista Cubana de Cirugía, 47(4), 1-7.

Rodriguez, C., Arce Aranda, C., Amarilla, L., Andreo, T., Araujo, D., Arzamendia, L., . . . Samaniego, C. (2013). Características clínicas y patológicas de los tumores de mediastino en un hospital universitario. Cir. Parag. Vol. 37; $N^{o}$ 2. Disponible en: http://scielo.iics.una.py/pdf/sopaci/v37n2/v37n2a06.pdf, 22-25. Obtenido de http://scielo.iics.una.py/pdf/sopaci/v37n2/v37n2a06.pdf

Salazar, C., Soto, L., Pucci, J. J., \& Brenes, F. (1980). Tumores primarios de Mediastino. Act. Méd. Cost. Volumen 23. $N^{\circ} 3 ., 253-265$.

Torres-Rodríguez, T., Herrera-Cruz, D., Gálvez-González, M., Moran-Ocaña, E., del CidHerrera, R. M., \& Gordillo-Castillo, R. (2016). Masas mediastinales: Epidemiología y decisiones estratégicas. Experiencia de 13 años. Neumología y cirugía de tórax, 75(4), $268-274$.

Tramontini, M. (27 de Abril de 2019). Madiastino. Obtenido de Universidad Nacional de La Plata. Facultad de Cierncias Medicas: http://www.anatomia-a.com.ar/MEDIASTINO.pdf

Vega Lorenzo, Y., Hidalgo Ávila, M., Martínez Lorenzo, F., García Martín, D., Aparicio Álvarez, F. E., \& González Díaz, E. D. (2016). Síndrome mediastinal. Presentación de un caso. MediCiego, 22(2), 61-67. 